\title{
TTR
}

Traduction, terminologie, re?daction

\section{Antoine Berman. Pour une critique des traductions : John Donne. Paris, Éditions Gallimard, " Bibliothèque des idées ", 1995, 278 pages.}

\section{Sherry Simon}

Volume 8, numéro 1, 1er semestre 1995

Orientations européennes en traductologie

URI : https://id.erudit.org/iderudit/037207ar

DOI : https://doi.org/10.7202/037207ar

Aller au sommaire du numéro

Éditeur(s)

Association canadienne de traductologie

ISSN

0835-8443 (imprimé)

1708-2188 (numérique)

Découvrir la revue

Citer ce compte rendu

Simon, S. (1995). Compte rendu de [Antoine Berman. Pour une critique des traductions : John Donne. Paris, Éditions Gallimard, «Bibliothèque des idées ", 1995, 278 pages.] TTR, 8(1), 282-287. https://doi.org/10.7202/037207ar

Tous droits réservés @ C TTR: traduction, terminologie, rédaction — Les auteurs, 1995 
Antoine Berman. Pour une critique des traductions: John Donne. Paris, Éditions Gallimard, «Bibliothèque des idées», 1995, 278 pages.

La lecture du livre posthume d'Antoine Berman est une expérience à la fois vivifiante et douloureuse. On est soulevé, comme toujours, par la passion et la justesse de sa parole; on est abattu par la 
conscience d'une œuvre trop tôt interrompue. Il n'y a aucun doute: l'apport de Berman à la vie des idées, à la réflexion sur la culture et la traduction, était unique. Il a inscrit la traduction au centre de la vie de la pensée, et son cuvre a eu un effet d'entraînement remarquable. L'Épreuve de l'étranger est devenu un livre de référence obligatoire, son titre presqu'un mot d'ordre dans la lutte contre l'ethnocentrisme culturel. Mais tout un ensemble très riche d'écrits (traductions et essais critiques), au cours d'une carrière trop courte, a propulsé la traduction vers l'avant-scène de la vie intellectuelle française, faisant la démonstration de la valeur heuristique de la pratique et de la théorie de la traduction.

Antoine Berman a consacré les derniers mois de sa vie, alors qu'il était terrassé par la maladie, à la rédaction de son dernier livre. Sa femme écrit: «Au cours des trois mois pendant lesquels a sévi sa brutale maladie, il a écrit ce livre, nuit et jour, sans relâche. A l'hôpital, il ne se couchait pas, transformant son lit en une étendue couverte de livres et de papiers». Malgré ces circonstances singulières, Pour une critique des traductions est un livre étonnamment solide et cohérent. Dans son introduction, Berman explique qu'il travaillait depuis des années à un livre sur la traduction en France du $14^{\mathrm{e}}$ au $17^{\mathrm{e}}$ siècle, mais qu'il avançait difficilement dans ce travail. Soudain, il lui est venu l'idée d'approfondir les nombreuses analyses de traductíon qu'il avait faites, notamment au cours de séminaires donnés au Collège international de philosophie, sentant qu'il y avait un lectorat qui attendait ce livre. C'est ainsi qu'il a pu très rapidement rassembler les analyses et les insérer dans un cadre d'une réflexion globale.

Il est question dans ce livre de créer une véritable critique des traductions. Non pas la critique qui se résume au jugement et à l'évaluation, mais une «analyse rigoureuse d'une traduction, de ses traits fondamentaux, du projet qui lui a donné naissance, de l'horizon dans lequel elle a surgi, de la position du traducteur» qui permet de dégager «la vérité d'une traduction» (pp. 13-14). Berman donne à la critique son sens le plus noble, et lui impose donc les exigences les plus rigoureuses. Les étapes du déroulement du livre sont faciles à résumer, d'autant plus que Berman lui-même fournit 
une synthèse de l'ouvrage dans son introduction. La première partie de l'ouvrage consiste en une critique des méthodes analytiques de Meschonnic d'une part, des approches «descriptives», «systémiques», "sémiotiques», de l'autre. Suivent une esquisse de la méthode proposée, et la démonstration, sous la forme d'une analyse des traductions de John Donne en français, en particulier celle d'un poème «Going to Sleep». Le but de Berman est d'établir les modalités d'une critique "productive» qui, même si elle donne un jugement négatif sur une traduction, éclaire «le pourquoi de l'échec traductif» et prépare «l'espace de jeu d'une retraduction» (p. 17).

Cette critique fait partie d'un ensemble de médiations qui comprend à la fois «translations non traductives» (tout le réseau d'échanges cognitifs et esthétiques qui construisent l'horizon de la traduction) et les traductions elles-mêmes.

Notons dans son introduction une affirmation intéressante et un peu énigmatique. Berman affirme avec force que la traduction est "chose romaine»: notre conception et pratique de la traduction est née à Rome. Il faut croire que cette affirmation s'inscrit en faux contre l'idée que la traduction naît dans la traduction biblique. Une note en bas de page critique la notion de «l'impensé théologique» de la traduction telle qu'elle est formulée notamment par Jean-René Ladmiral.

Qu'est-ce que la critique? Quiconque s'est déjà engagé dans la tâche de lire, d'évaluer et de déterminer l'apport d'une traduction en tant que traduction sait à quel point ce travail est difficile. Peu de théoriciens ont réussi jusqu'ici à imposer des modèles heuristiques éclairants et surtout généralisables. Berman cite en exemple d'abord le travail d'Henri Meschonnic, auquel il accorde un grand mérite, mais dont il souligne le ton toujours très négatif et destructeur. Pour ce qui est des praticiens de la théorie du polysystème comme Gidéon Toury et Annie Brisset, Berman leur reproche l'attitude déterministe et fonctionnaliste qui empêche de questionner le pourquoi d'une traduction; et qui aboutit à «une négation du rôle créateur et autonome du traduire dans l'histoire 
occidentale» (p. 54). Berman souligne bien entendu la force et la rigueur du travail de ces analystes, ne mettant pas en question le bond en avant que cette approche a permis aux études traductologiques. Mais il insiste sur les limites d'un modèle déterministe.

L'autre objection qu'il soulève contre l'une des thèses importantes de l'école de Tel-Aviv me semble, par contre, plus discutable. Berman soutient que «les littératures étrangères traduites ne s'intègrent généralement pas à la littérature autochtone», sauf dans le cas de très grandes traductions; elles restent des «littératures étrangères». Cette perception me paraît bien «hexagonale», et pas du tout généralisable à l'ensemble des relations littéraires.

Dans l'exposé de sa méthode d'analyse, Berman essaie d'élaborer un vocabulaire qui pourrait nous aider à comprendre ce qu'est la «lecture» d'une traduction. On reconnaît ici le Berman qui dans «la Traduction et la lettre, ou l'auberge du lointain» (dans les Tours de Babel, 1985) essaie de saisir les mécanismes intimes du processus traductionnel. Mais dans ce cas-ci il est question de saisir la trame vivante mais peu conceptualisée de la lecture des traductions. Cette lecture du texte traduit découvre des «zones textuelles» défectueuses, problématiques mais aussi des zones «que je qualifierai de miraculeuses, en ceci qu'on se trouve en présence non seulement de passages visiblement achevés, mais d'une écriture qui est une écriture-de-traduction, une écriture qu'aucun écrivain français n'aurait pu écrire, une écriture d'étranger harmonieusement passée en français, sans heurt aucun (ou, s'il y a heurt, un heurt bénéfique)» (p. 66). De la même manière, la relecture de l'œuvre originale permettra de découvrir «ces passages de l'original qui, pour ainsi dire, sont les lieux où elle se condense, se représente, se signifie ou se symbolise. Ces passages sont les zones signifiantes où une œuvre atteint sa propre visée (pas forcément celle de l'auteur) et son propre centre de gravité. L'écriture y possède un très haut degré de nécessité» (p. 70). Berman donne donc une importance capitale à la capacité analytique du critique, mais le critique doit aussi comprendre ce que Berman appelle la "position traductive» du 
traducteur, son projet de traduction et son horizon traductif (pp. 7475).

Pour ce qui est de l'analyse des traductions à proprement parler, Berman propose deux critères: la poéticité et l'éthique. Pour ce qui est de la poéticité (l'obligation pour le traducteur de "faire œuvre»), cela semble aller de soi. Par contre, quant à l'éthique, je crois lire un renversement important par rapport à la conclusion très ferme de l'Épreuve de l'étranger. Au lieu de mettre l'accent sur le devoir à l'égard de «la lettre», ici Berman dira que «[1]e traducteur a tous les droits dès qu'il joue franc jeu» (p. 93). Le devoir du traducteur n'est pas à définir par une obligation prédéterminée vis à vis du texte mais bien dans la transparence de cette relation, quelle qu'elle soit. Berman abandonne les débats futiles entre sourciers et ciblistes pour insister davantage sur l'importance du projet du sujet traduisant. Pour ma part, je suis tout à fait d'accord avec cette mise au point, d'autant plus que la deuxième partie du livre va montrer que cette position ne mène absolument pas à l'acceptation béate de tout projet traductionnel, mais impose un devoir de cohérence qui n'est pas nécessairement facile à atteindre.

La deuxième partie du livre est donc consacrée à une démonstration de la méthode de Berman; elle consiste en une critique de la traduction de la poésie de John Donne (et surtout un poème, "Going to Bed») en français. Il suffit de dire ici que Berman n'apprécie pas du tout la traduction publiée par Gallimard par J. Fuzier et Y. Denis en 1962 (et rééditée en 1991), et il en fait la démonstration de manière convaincante, tout en évoquant l'ensemble des éléments qui ont créé et qui pourraient recréer le Donne français. Les paramètres de la discussion sont vastes; Berman nous invite à pénétrer un monde aéré et passionnant. Une seule lecture ne permet pas de saisir l'ensemble des éléments de la discussion, ni de juger de la pertinence de chacun des commentaires de Berman. Mais il est évident que cet exposé servira de modèle à une critique qui refuse une conception simpliste des «normes» translationnelles et qui donne au traducteur la pleine responsabilité de sa relative liberté. 
Pour une critique des traductions n'est pas un manuel, mais sa valeur pédagogique est évidente. Autant les chercheurs accomplis que les étudiants y trouveront leur compte. Les lecteurs enthousiastes de ce livre se réjouiront de la notice en fin de livre des titres «à paraitre» d'Antoine Berman: Jacques Amyot, traducteur français et Traduire Benjamin.

Sherry Simon Université Concordia 